\title{
A longitudinal study of brain atrophy and cognitive disturbances in the early phase of relapsing-remitting multiple sclerosis
}

\author{
R Zivadinov, J Sepcic, D Nasuelli, R De Masi, L Monti Bragadin, M A Tommasi, \\ S Zambito-Marsala, R Moretti, A Bratina, M Ukmar, R S Pozzi-Mucelli, A Grop, \\ G Cazzato, M Zorzon
}

Department of Clinical Medicine and Neurology, University of Trieste, Trieste, Italy

R Zivadinov

D Nasuelli

R De Masi

L Monti Bragadin

M A Tommasi

S Zambito-Marsala

R Moretti

A Bratina

G Cazzato

M Zorzon

Department of

Radiology

M Ukmar

R S Pozzi-Mucelli

Department of Electrical, Electronics and Computer Science A Grop

Neurological Clinic, University of Rijeka, Rijeka, Croatia

J Sepcic

Correspondence to:

Dr R Zivadinov,

Neurological Clinic,

Cattinara Hospital, Strada di

Fiume 447, 34149 Trieste,

Italy

zivadinov@hotmail.com.

Received 15 August 2000 and in final form

27 November 2000

Accepted 14 December 2000

\begin{abstract}
Objective-(a) To establish whether the cognitive decline of the early phase of relapsing-remitting multiple sclerosis depends on the progression of the burden of disease, or on the loss of brain parenchyma, or is influenced by both; $(b)$ to monitor the loss of brain parenchyma in the early phase of the disease; and (c) to examine its possible relation with the progression of physical disability.

Methods-For 2 years 53 patients with clinically definite relapsing-remitting multiple sclerosis with disease duration 1-5 years and expanded disability status scale $\leqslant 5.0$ at baseline were monitored. The neuropsychological performances, the psychological functioning, the neurological impairment, and the disability have been assessed at baseline and after 2 years. Patients also underwent PD/T2 and T1 weighted brain MRI. T2 and T1 lesion volumes were measured by a semiautomatic technique. Quantification of brain parenchymal volumes was obtained using a highly reproducible computerised interactive program. The relation between cognitive impairment and MRI findings has been investigated by partial correlation and stepwise multiple regression analyses excluding the effects of age, education, anxiety, depression, and total days of steroid use.
\end{abstract}

Results-In the 2 years of the study the mean change for $T 2$ and $T 1$ lesion volumes and brain parenchymal volumes were +1.7 $\mathrm{ml}(95 \%$ confidence interval $(95 \% \mathrm{CI}) \mathbf{1 . 3 -}$ 2.2, p $=0.005,(29.8 \%)$; $+0.2 \mathrm{ml}, 95 \%$ CI 0.15-0.26, p=0.004, (25\%); and $-32.3 \mathrm{ml}$, $95 \%$ CI $24.2-42.3$, p $<0.0001,(2.7 \%)$, respectively. Overall, 14 patients (26.4\%) were judged to be cognitively impaired at baseline and $28(52.8 \%)$ at the end of the follow up. Of the 18 neuropsychological tests and subtests employed in the study, patients with multiple sclerosis failed $\mathbf{5 . 8}$ (SD 2.3) tests at the baseline and 8.4 (SD 2.9) $(p<0.0001)$ tests at the end of the study. When the cognitive changes were examined in individual patients, five $(9.4 \%)$ of them were considered cognitively improved, $33(62.3 \%)$ remained stable, and $15(28.3 \%)$ worsened over 2 years. $T 2$ and $T 1$ volume changes in improved, stable, and worsened patients did not show any significant difference, whereas brain parenchymal volume decrease in cognitively worsened patients was significantly greater $(-66 \mathrm{ml}(5.4 \%)$, 95\% CI 37-108.9, $\mathrm{p}=0.0031)$. The cognitive impairment was independently predicted over 2 years only by the change of brain parenchymal volumes $(R=0.51, p=0.0003)$. Ten patients $(18.9 \%)$, who worsened by one or more points in the EDSS during the follow up period had significant decreases in brain parenchymal volumes (-99 $\mathrm{ml}(8 \%), 95 \%$ CI 47.6-182.3, $\mathrm{p}=0.005)$. At the end of the study the loss of brain parenchyma correlated significantly with change in EDSS $(r=0.59, \mathrm{p}<0.0001)$.

Conclusions-In the early phase of relapsing-remitting multiple sclerosis the cognitive deterioration relies more on the development of brain parenchymal volume atrophy than on the extent of burden of disease in the brain. The loss of brain parenchymal volume underlies the progressive accumulation of physical disability from the initial phase of the disease, which becomes more demonstrable only if studied with longer observation periods. Probably, the main pathological substrate of brain atrophy in the early stage of the disease is early axonal loss, which causes the progression of neurological deficits and the development of cognitive impairment. These data support the debated opinion that disease modifying therapy should be initiated as early as possible. (f Neurol Neurosurg Psychiatry 2001;70:773-780)

Keywords: multiple sclerosis; cognitive dysfunction; brain atrophy

Different MRI techniques for monitoring disease evolution in patients with multiple sclerosis have been widely employed in therapeutic clinical trials. ${ }^{1-4}$ Protocols for MRI are now routinely incorporated in such studies, providing powerful evidence of a therapeutic effect and extending clinical findings. Conventional and non-conventional MRI techniques have also been used to demonstrate a relation between MRI abnormalities and cognitive dysfunction in many cross sectional studies. ${ }^{5-18}$

Only a few longitudinal studies examined the correlation between MRI abnormalities and patterns of cognitive impairment. ${ }^{5}{ }^{19-21}$ None of these studies involved patients with multiple sclerosis in the early stages of the disease and 
most of them used only conventional MRI techniques and qualitative or manual quantification postimaging analysis methods. Data from more recent studies indicate that cognitive disturbances can be present early in the clinical evolution of the disease but also that they may not be obvious. ${ }^{22} 23$

Recently, several cross sectional studies demonstrated that the pathogenesis of cognitive impairment in patients with multiple sclerosis does not depend only on the extent and severity of the pathological process within brain lesions, but also on the microscopic pathological changes affecting the normal appearing brain tissue..$^{7-1118}$ In fact, the new technologies employed in pathological and MRI studies showed that progressive pathological changes occur far earlier in the disease process than previously thought, and that widespread abnormalities can develop early in the brain parenchyma..$^{24-31}$

The recent demonstration of early irreversible axonal transection ${ }^{25}$ in multiple sclerosis led us to conduct a longitudinal study designed to monitor the cognitive performances and the changes shown by different conventional and non-conventional MRI techniques in 53 patients with early phase relapsing-remitting multiple sclerosis over 2 years. The aim of the study was to establish whether the cognitive decline depends on the progression of the burden of disease, or on the loss of brain parenchyma, or it is influenced by both.

Relatively few longitudinal natural history MRI studies of cerebral atrophy in multiple sclerosis have been reported to date. ${ }^{32-35}$ None of these studies evaluated the progression of cerebral atrophy in the early phase of relapsingremitting disease. So, the secondary objective of our study was to monitor the loss of brain parenchyma in the early phase of the disease and to test its relation with the progression of physical disability.

\section{Material and methods \\ PATIENTS}

Eighty three consecutive unselected patients affected by definite multiple sclerosis according to the diagnostic criteria proposed by Poser et $a l{ }^{36}$ participated in the study. The study involved patients who were being seen in routine follow up, or for problems not related to cognitive disturbances or disease exacerbation.

To assess the attention and the compliance to attend the proposed battery of tests and the ability to follow the scheduled protocol for 2 years, we decided to screen our multiple sclerosis population with two tests before the complete evaluation of their neuropsychological capacities. The screening was performed using the mini mental state examination $(\mathrm{MMSE})^{37}$ and the standard Raven progressive matrices (SRPM) ${ }^{38}$ adjusted for age, sex, and educational level. Arbitrarily we decided to choose a cut off value of $\geqslant 24$ for MMSE and the 15th percentile for SPRM. Inclusion criteria were definite multiple sclerosis, relapsingremitting disease course, age 18-60 years, disease duration $1-5$ years, EDSS score $\leqslant 5.0,{ }^{39}$ and written informed consent from all the patients. Exclusion criteria were MMSE score $<24$; SRPM $<15$ th percentile; concomitant disorders (cerebrovascular disease, neurodegenerative disorder, positive history of alcohol misuse, severe visual deficit, or severe upper limb motor impairment), or pre-existing psychiatric or psychological disorders that, themselves, can cause cognitive dysfunction or can impede the completion of the cognitive tests; concurrent exacerbation of the disease; and immunomodulating, steroid, or psychoactive drug treatment in the 3 months preceding the entry to the study. During the follow up period the patients with multiple sclerosis were checked for the appearance of concomitant disorders which could influence the cognitive performances. During the study period, treatment with disease modifying therapy was not used in any enrolled patient. Corticosteroid administration was allowed in cases of exacerbation at the discretion of each patient's neurologist.

A neurologist (RZ) specialised in multiple sclerosis care classified patients as having relapsing-remitting multiple sclerosis according to the disease course criteria recently published by Lublin and Reingold. ${ }^{40}$ The exacerbations were defined as the appearance or reappearance of one or more symptoms attributable to multiple sclerosis, accompanied by objective deterioration lasting at least 24 hours on neurological examination, in the absence of fever, and preceded by neurological stability for at least 30 days and in the absence of steroid withdrawal within 60 days of the new event.

Thirty patients (19 women and 11 men) were excluded from the study. Reasons of exclusion were secondary or primary chronic progressive disease course (eight patients), IQ less than the 15th percentile on SRPM adjusted for age, sex, and educational level (five patients), severe visual deficits or severe upper limb motor impairment (four patients), steroid treatment in the preceding 3 months (four patients), history of alcohol misuse (three patients), immunomodulating drug treatment in the preceding 3 months (two patients), psychoactive drug treatment in the preceding 3 months (two patients), MMSE<24 (one patient), refusal to participate in the study (one patient). Hence, the remaining 53 patients, 37 $(69.8 \%)$ women and $16(30.2 \%)$ men, participated in the present study.

Our data could not represent the real prevalence of cognitive disturbances in the early phase of relapsing-remitting multiple sclerosis because we choose to screen our population with MMSE and SPRM before inclusion in the study to check their attention, compliance, and ability to sustain a 2 year follow up study.

All patients had a full neurological examination at baseline and at the end of follow up. The neurological impairment, the disability, and the independence of the patient have been measured using the EDSS and the functional independence measure (FIM). ${ }^{41}$ At the end of the study the patients were classified as clinically worsened if they decreased at least 1.0 EDSS score point from the baseline status. 
Table 1 Neuropsychological scores of patients with relapsing-remitting multiple sclerosis at baseline and at the end of follow up testing. The cut off scores indicating impairment are provided

\begin{tabular}{|c|c|c|c|c|c|}
\hline & $\begin{array}{l}\text { Cut off } \\
\text { scores }\end{array}$ & $\begin{array}{l}\text { Baseline } \\
\text { (53 patients) } \\
\text { mean (SD) }\end{array}$ & $\begin{array}{l}\text { Follow up } \\
\text { ( } 53 \text { patients) } \\
\text { mean (SD) }\end{array}$ & $\begin{array}{l}\text { Mean \% change } \\
(95 \% \mathrm{CI})\end{array}$ & p Value \\
\hline Stroop colour word interference test $(\mathrm{ms})$ & $>69$ & $71.3(5.4)$ & $81.9(9.4)$ & $14.9(11.2-19.5)$ & $<0.0001$ \\
\hline Paced auditory serial addition test & $<48$ & $47.9(11.1)$ & $42.3(15.7)$ & $11.7(8.8-15.3)$ & 0.035 \\
\hline Standard Raven progressive matrices & $>110$ & $119.5(13.7)$ & $113.8(13)$ & $4.8(3.6-6.3)$ & 0.033 \\
\hline \multicolumn{6}{|l|}{ Wechsler adult intelligence scale } \\
\hline Verbal fluency (total No) & $<34$ & $36.7(9.5)$ & $33.1(10.3)$ & $9.8(7.3-12.8)$ & 0.064 \\
\hline Digit span forward & $<5$ & $5.1(1)$ & $4.4(1.3)$ & $13.7(10.3-17.9)$ & 0.003 \\
\hline Digit span feedback & $<4$ & $2.8(1.1)$ & $2.8(0.8)$ & $0(-)$ & 0.839 \\
\hline Similarities (No correct) & $<11$ & $8.3(2.3)$ & $7.1(1.5)$ & $14.5(10.9-19)$ & 0.002 \\
\hline Cube subtest & $<10$ & $8.3(1.5)$ & $7.5(1.3)$ & $9.6(7.2-12.6)$ & 0.003 \\
\hline \multicolumn{6}{|l|}{ Bilingual aphasia test } \\
\hline Story recall test (No of errors) & $>2$ & $1.1(0.9)$ & $1.5(1.6)$ & $36.4(27.2-47.7)$ & 0.099 \\
\hline Mental arithmetic (No of errors) & $>2$ & $1.5(1.7)$ & $2.7(2.4)$ & $80(60-104.8)$ & 0.048 \\
\hline Morphology (No of errors) & $>5$ & $2.9(3.5)$ & $4(3.2)$ & $37.9(28.4-49.6)$ & 0.091 \\
\hline Syntax (No of errors) & $>14$ & $9.3(6.8)$ & $14.1(11)$ & $51.6(38.6-67.6)$ & 0.008 \\
\hline Semantic (No of errors) & $>7$ & $3.5(3.3)$ & $4.7(3.3)$ & $34.3(25.7-44.9)$ & 0.05 \\
\hline Syntactic comprehension (No of errors) & $>12$ & $14(9.1)$ & $15(10.3)$ & $7.2(5.4-9.4)$ & 0.590 \\
\hline \multicolumn{6}{|l|}{ Spontaneous speech (No of errors) } \\
\hline Verbal paraphasias & $>1$ & $1(1)$ & $3.7(1.5)$ & $270(202.2-353.7)$ & $<0.0001$ \\
\hline Agrammatisms & $>2$ & $3.6(3.2)$ & $4(2)$ & $11.1(8.3-14.5)$ & 0.489 \\
\hline Neologisms & 0 & $0.3(0.9)$ & $0.1(0.3)$ & $66.6(49.9-87.2)$ & 0.402 \\
\hline Mini mental state examination & $>24$ & $29.4(1)$ & $29.2(1.4)$ & $0.68(0.51-0.89)$ & 0.366 \\
\hline Hamilton depression rating scale & & $5.6(6.7)$ & $6.4(7)$ & $14.2(10.7-18.6)$ & 0.574 \\
\hline Hamilton anxiety rating scale & & $21.4(22.2)$ & $22.9(21.2)$ & $7(5.2-9.2)$ & 0.727 \\
\hline
\end{tabular}

Comparison between the baseline and the follow up was performed by paired $t$ test.

NEUROPYCHOLOGICAL EVALUATION

Within 48 hours of MRI examination, the same extensive neuropsychological battery of tests used at baseline was readministered to the patients at the end of the 2 year follow up. The battery comprised measures of attention/ concentration/information processing speed, memory function, abstract/conceptual reasoning, visuoperceptual skills, verbal intelligence, and language skills. We specifically chose tests that do not require fine visual acuity or motor speed/dexterity.

The attention/concentration/information processing speed was assessed by the 3 second performance of the paced auditory serial addition test (PASAT), ${ }^{42}$ the colour/word-word conditions of the Stroop colour word interference test $(\mathrm{STROOP})^{43}$ and the subtest of the Wechsler adult intelligence scale-revised (WAIS-R; verbal fluency $)^{44}$. Memory was tested by the two subtests of the WAIS-R (digit span forward and feedback) and by the subtest of the Bilingual aphasia test (BAT; story recall test ${ }^{45}$. Abstract/ conceptual reasoning skills were evaluated by SRPM. Visuoperceptual skills were evaluated using the cube subtest of the WAIS-R. Verbal intelligence was assessed by means of the similarities subtest of the WAIS-R and the mental arithmetic subtest of the BAT. The linguistic parameters chosen for the evaluation were syntactic comprehension of mistakes, semantic mistakes, and syntactical and morphological mistakes. The numbers of verbal paraphasias, neologisms, and agrammatisms as a measure of linguistic property and ability were assessed by means of the BATs. These tests are routinely used in the assessment of cognitive impairment in multiple sclerosis and are extensively described elsewhere. ${ }^{46-48}$

At the baseline and at the end of the follow up, the number of failed tests was calculated for each patient. According to previously published criteria ${ }^{49}$ established cut off scores to indicate impairment (table 1) have been used. Patients have been considered affected by overall cognitive impairment if they obtained abnormal results in two or more cognitive domains. At the end of the study we decided to classify the patients according to their cognitive status as follows:

(a) Cognitively improved patients-Patients who presented more than $25 \%$ of improvement from the baseline status in the neuropsychological tests exploring at least two or more cognitive domains, and who simultaneously had not worsened more than $25 \%$ in the tests exploring other cognitive domains.

(b) Cognitively worsened patients-Worsening required a $25 \%$ decline from baseline tests scores exploring at least two or more cognitive domains, without $25 \%$ simultaneous improvement in the tests exploring other cognitive domains.

(c) Cognitively stable patients-Patients not meeting the above mentioned criteria were classified as stable.

This classification, used also in a longitudinal study performed by Hohol et al had been chosen so that each patient could serve as his or her own comparison and control for effects of age, sex, and education. The requirement that at least two changes in cognitive domains by $25 \%$ from baseline status was based on the results of previous studies in the early phase of multiple sclerosis ${ }^{50} 51$

The Hamilton depression rating scale $(\mathrm{HDRS})^{52}$ and the Hamilton anxiety rating scale (HARS $)^{53}$ were employed, after a psychiatric interview with the patient to exclude psychological disorders which can contribute to cognitive disturbances, and were repeated at the end of the follow up.

\section{MAGNETIC RESONANCE IMAGING}

Image acquisition

Brain MRI was performed at baseline and at the end of the follow up on a Philips Gyroscan S 15 ACS II 1.5 tesla unit. Axial images of the brain were obtained with $5 \mathrm{~mm}$ slice thickness using PD/T2 weighted SE sequences (TR 
2709/TE 20-80) and unenhanced T1 weighted CSE sequences (TR 600/TE 27). A matrix of $179 \times 256$ pixels has been used for a total of 24 sections. Field of view was $220 \mathrm{~mm}$. Patients were positioned in the magnet according to European Community guidelines. ${ }^{54}$

\section{Image analysis}

Two investigators, blinded to the patient's clinical characteristics and the cognitive tests results (RDM and ND), performed the image analysis at baseline and at the end of the study on a Sun Ultra 5 Promo workstation (Sun Microsystems, Mountain View, CA, USA). Imaging data have been transferred directly to the computer system of the medical imaging processing group by a home developed network transferring system. Lesion load was calculated using a highly reproducible local thresholding technique for lesion segmentation..$^{55}$ The lesions were first outlined on PD weighted hard copies on each axial slice (T2 weighted scans were always used to increase confidence in lesion detection). The measurements of lesion area were then performed on computer displayed images by the same observers, keeping the marked hard copies as a reference. A conservative approach for the calculations of lesions in $\mathrm{T} 1$ weighted images was used. A hypointense lesion was defined as any region visible on the $\mathrm{T} 1$ weighted sequence with a low signal intensity between those of the $\mathrm{CSF}$ and grey matter and corresponding to a region of high signal intensity on the T2 weighted sequence. Lesions were delineated as regions of interest (ROIs) and the volume was simply calculated for each sequence by multiplying the total ROI area by the slice thickness. The results are expressed in $\mathrm{ml}$.

The evaluation of brain atrophy was performed on T1 weighted CSE sequences measuring the brain parenchymal volumes. An interactive home developed program which incorporates semiautomatic and automatic segmentation processes was used for the measurements. The whole brain parenchyma volume was analysed (from the cerebral hemispheres and the other supratentorial structures to the cerebellum and the brain stem). A stereoatactic atlas was used as a reference for segmenting the different brain structures. Firstly, the external edge of whole brain parenchyma was determined by semiautomatic iterative morphological scalping of the external brain surface. Then, the automatic segmentation of the CSF spaces and brain parenchyma was performed using a knowledge based automatic segmentation algorithm for histogram thresholding analysis. This segmentation process automatically creates brain parenchyma only ( $1 \mathrm{~A}$ and $\mathrm{C}$ ) and CSF only (fig $1 \mathrm{~B}$ and $\mathrm{D}$ ) images and calculates the volumes for brain parenchyma and CSF. To determine the reproducibility of the method, 10 patients with multiple sclerosis and 10 sex and age matched healthy volunteers had two separate MR scans within 1 week. Reproducibility was calculated as a coefficient of variation $(\mathrm{CV})(100 \% \times \mathrm{SD} /$ mean) between the repeated measurements, by two investigators blinded to patient details and diagnosis. The mean CV for brain parenchymal volumes in this group was $0.39 \%$ (range 0.09 0.56 ) for between observer reproducibility and $0.37 \%$ (range $0.09-0.52$ ) for within observer reproducibility. This clearly indicates the extremely high precision of the segmentation method.

STATISTICAL ANALYSIS

Statistical analysis was performed with the Statistical Package for the Social Sciences (SPSS, version 10.0). For comparisons between groups the $\chi^{2}$ test, paired $t$ test, and analysis of variance (ANOVA) were used, as appropriate. Cognitively improved, stable, and worsened patients with multiple sclerosis at the end of the follow up have been compared using ANOVA adjusted for age, sex, years of education, anxiety, depression, and total days of steroid use. The relation between cognitive impairment and MRI changes at baseline and at the end of the follow up has been investigated by partial correlation coefficient analysis corrected for multiple comparisons and by multivariate stepwise linear regression analysis (backward selection model) excluding the effects of age, education, anxiety, depression, and total days of steroid use. For the correlation and multiple regression analyses we created an overall cognitive deterioration composite score from the 18 neuropsychological tests employed. For each patient, the results from all the neuropychological tests were scored using a standardised method ${ }^{49}$ based on a comparison with the percentile distribution of values in a large group of Italian subjects. These scores ranged from 0 to 4 , where grade 0 means a very poor performance and grade 4 means a normal performance. Individual test scores were then summed to provide an overall cognitive deterioration composite score for each patient. The minimum significance level for entry and for staying in the equation was 0.05. All $\mathrm{p}$ values were based on two tailed tests.

\section{Results}

The study consisted of 53 patients ( 37 women and $16 \mathrm{men}$ ) affected by the relapsingremitting form of multiple sclerosis. Mean age, disease duration, and educational level at baseline were 30.2 years (SD 9.4; range 21-58 years), 3.8 years (SD 1.3; range $1-5$ years), and 11.3 years (SD 3.2; range 8-17 years), respectively. Mean annual relapse rate over the 2 years before the patients' entrance in the study was 1.0 (SD 1.8; range 0-6). Mean EDSS score at baseline was 1.6 (range 0-5.0), median EDSS score at baseline was 1.0. Mean FIM score at baseline was 125.7 (range 121-126), median FIM score at baseline was 126 . At the end of the follow up period we did not find significant differences from baseline data for any of the clinical variables, except for disability (mean EDSS 1.8, median EDSS 1.5, $\mathrm{p}=0.007)$. Total days of steroid use during the follow up was 9.7 (SD 6.5; range 5-16).

Initial and follow up neuropsychological performances are listed in table 1. Overall, 14 patients $(26.4 \%)$ were judged as cognitively 
impaired at baseline and $28(52.8 \%)$ at the end of the follow up. Of the 18 neuropsychological tests and subtests employed in the study, patients failed 5.8 (SD 2.3; range 1-12) at baseline and 8.4 (SD 2.9; range 3-15), at the end of the follow up $(\mathrm{p}<0.0001)$. When the cognitive changes were examined in individual patients, five $(9.4 \%)$ were considered cognitively improved, $33(62.3 \%)$ remained stable, and $15(28.3 \%)$ worsened after the 2 year folworsened patients were studied according to find any significant difference between the three groups except for the educational level, which was higher in the cognitively improved group $(p=0.018)$. Compared with the baseline status the patients did not present significant changes for depression and anxiety at the end of the study.

Baseline loads of multiple sclerosis lesions visible on T2 and T1 weighted scans, baseline brain parenchymal volumes, and changes in these MRI parameters over 2 years are shown low up. The cognitively improved, stable, and the demographic and clinical data. We did not

in table 2. During the follow up period the patients showed a significant increase in lesion loads and a decrease in brain parenchymal volumes, which was more evident for loss of brain parenchyma $(\mathrm{p}<0.0001)$. Changes inT2 and $\mathrm{T} 1$ volume in improved, stable, and worsened patients did not show any significant difference, whereas the decrease in brain parenchymal volume in cognitively worsened patients was significantly higher (-66 ml (5.4\%), 95\% CI $37-108.9 ; \mathrm{p}=0.0031)$. A correlation analysis performed to verify whether the loss of brain parenchymal volume in cognitively worsened patients was age dependent failed to show a relation between these two variables $(r=0.22$, $\mathrm{p}=0.513$ ).

The 10 patients (18.9\%), who worsened by on or more points in the EDSS during the follow up period, had a significant decrease in brain parenchymal volumes (-99 $\mathrm{ml}(8 \%)$, 95\% CI 47.6-182.3; $\mathrm{p}=0.005)$. The change in disability rate was not significant for changes in $\mathrm{T} 2$ and $\mathrm{T} 1$ lesion volumes over the 2 years.
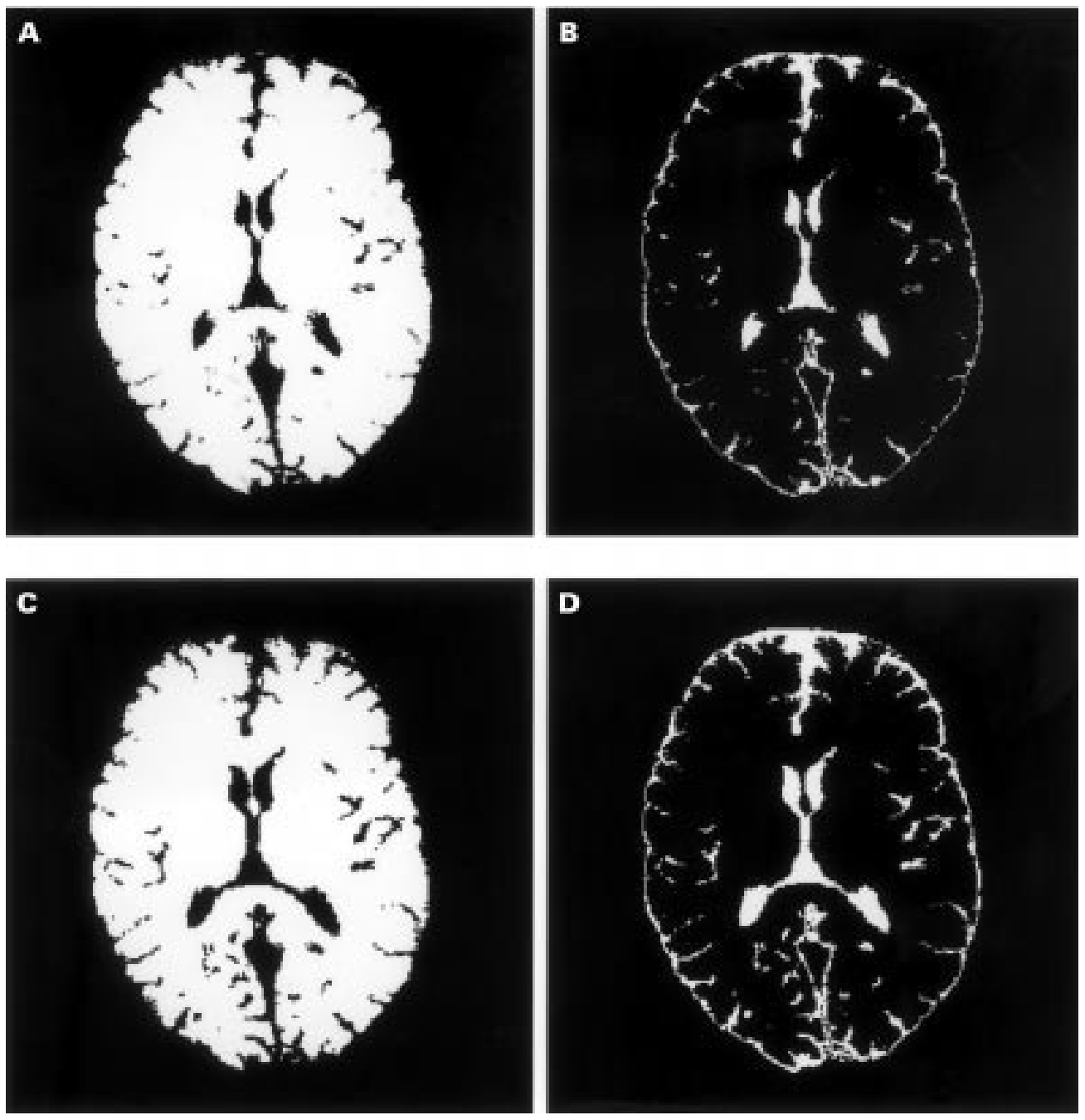

Figure 1 Brain parenchyma only ( $A$ and $C$ ) and CSF only ( $B$ and $D)$ images in a 32 year old man with early relapsing-remitting multiple sclerosis ( $A$ and $B$ show baseline acquisition and $C$ and $D$ acquisition at study completion) who worsened in his cognitive performance over 2 years of follow up. The loss of brain parenchyma, as reflected by the enlargement of ventricles and sulci was $4.3 \%$. All the patients completed the designed protocol for clinical, neuropsychological, and radiological testing in the follow up period. Only two patients had their MRI examination at the end of the study 48 hours after the neuropsychological testing. Additional MRI examination were performed on some patients at the time of clinical exacerbation, but these examinations were not taken into account in the follow up study. 
Table 2 Baseline T2 and T1 lesion loads and brain parenchymal $(B P)$ volumes and changes over 2 years in patients with relapsing-remitting multiple sclerosis

\begin{tabular}{|c|c|c|c|}
\hline & $\begin{array}{l}\text { Baseline } \\
\text { (53 patients) }\end{array}$ & $\begin{array}{l}\text { Follow up } \\
\text { (53 patients) }\end{array}$ & $p$ Value \\
\hline T2 lesion load (ml): & & & 0.005 \\
\hline Mean (SD) & $5.7(5.9)$ & $7.4(7)$ & \\
\hline Range & $0.02-29.9$ & $0.23-27$ & \\
\hline \multicolumn{4}{|l|}{ T2 change over 2 y: } \\
\hline $\begin{array}{l}\text { Mean (\%) } \\
(95 \% \mathrm{CI})\end{array}$ & & $\begin{array}{l}+1.7(29.8) \\
(1.3-2.2)\end{array}$ & \\
\hline $\mathrm{T} 1$ lesion load (ml) & & & 0.004 \\
\hline Mean (SD) & $0.8(1.1)$ & $1(1.1)$ & \\
\hline Range & $0-5.3$ & $0-5.3$ & \\
\hline \multicolumn{4}{|l|}{$\mathrm{T} 1$ change over $2 \mathrm{y}$ : } \\
\hline $\begin{array}{l}\text { Mean (\%) } \\
(95 \% \mathrm{CI})\end{array}$ & & $\begin{array}{l}+0.2(25) \\
(0.15-0.26)\end{array}$ & \\
\hline $\mathrm{BP}$ volume (ml) & & & $<0.0001$ \\
\hline Mean (SD) & $1219.1(76.8)$ & $1186.8(80.7)$ & \\
\hline Range & $\begin{array}{l}1054.1- \\
1306.3\end{array}$ & $981.9-1289.2$ & \\
\hline \multicolumn{4}{|l|}{$\mathrm{BP}$ change over $2 \mathrm{y}$ : } \\
\hline Mean (\%) & & $-32.3(2.65)$ & \\
\hline$(95 \% \mathrm{CI})$ & & $(24.2-42.3)$ & \\
\hline
\end{tabular}

Comparison between the baseline and the follow up was performed by paired $t$ test.

Overall cognitive impairment did not correlate significantly with any of the demographic, clinical, or MRI variables at baseline. At the end of the follow up period only the decrease in brain parenchymal volumes was significantly related to changes in cognitive performances $(r=0.31, \mathrm{p}=0.0031)$. Brain parenchymal volume correlated significantly with $\mathrm{T} 2$ and $\mathrm{T} 1$ lesion loads at baseline $(r=0.33, \mathrm{p}=0.021$ and $r=0.37, \mathrm{p}=0.008$ ), respectively. The relation between the changes in these variables became more robust after 2 years $(r=0.61, \mathrm{p}<0.0001$ and $r=0.78$, respectively, $\mathrm{p}<0.0001)$. Moreover, at the end of the study the change of brain parenchymal volume correlated significantly with changes in EDSS $(r=0.59, \mathrm{p}<0.0001)$, FIM ( $r=0.33, \mathrm{p}=0.022)$, and, weakly, with annual relapse rate over 2 years $(r=-0.29$, $\mathrm{p}=0.043)$. Other significant correlations were detected at the end of the follow up period between changes in $\mathrm{T} 1$ lesion load, changes in EDSS, and duration of disease $(r=0.47$, $\mathrm{p}=0.001$ and $r=0.29, \mathrm{p}=0.045)$, respectively, and between changes in T2 lesion load and changes in EDSS ( $r=0.38, \mathrm{p}=0.007)$.

We developed several multiple regression models using the overall cognitive deterioration and the brain parenchymal volume as dependent variables. The cognitive impairment was independently predicted over 2 years only by the mean absolute change of brain parenchymal volumes $(R=0.51, \mathrm{p}=0.0003)$. The loss of brain parenchyma was independently correlated with T1 lesion load at baseline $(R=0.59$, $\mathrm{p}<0.0001$ ) and to the mean absolute change of T1 lesion load and the change in EDSS at the end of the follow up $(R=0.69, \mathrm{p}<0.00001$ and $R=0.81, \mathrm{p}<0.0001$, respectively).

\section{Discussion}

To the best of our knowledge this is the first longitudinal study of brain atrophy and cognitive performances conducted on a homogeneous group of patients not treated with disease modifying agents in the early phase of relapsing-remitting multiple sclerosis.

Few longitudinal studies have explored the relation between the cognitive status and the
MRI parameters. These studies ${ }^{19-21}$ used inaccurate postimaging analysis techniques to measure the burden of disease and did not focus attention on the microscopic damage of brain parenchyma. Only one longitudinal correlation study using non-conventional MRI techniques, has been carried out. ${ }^{5}$

Our study, performed on relatively young patients with short disease duration, demonstrated that a remarkable part of the study population had global cognitive worsening. These data suggest that cognitive decline occurs early in the course of the disease and that over 2 years the patients can develop a sustained progression of the impairment of their cognitive performances.

Some patients improved in their follow up neuropsychological performances. As the same tests were used at the baseline and at the end of the follow up, a potential learning effect which may also have masked a more severe cognitive deterioration over time in the remaining patients can be hypothesised. In our opinion however, the complexity of the battery of tests used and the period of follow up, too long to show "imprinting" capacities of memory reinforcement in execution of tests, make unlikely a lasting effect of practice. ${ }^{57}$

Compared with the cognitively improved and stable patients, the 15 patients considered cognitively worsened at the end of the study showed a more conspicuous decrease of brain parenchymal volumes than an increase of T2 and T1 lesion loads. These data, supported by the findings of Hohol et a $\bar{l}$ who demonstrated, in a heterogeneous group of patients with multiple sclerosis during a 1 year follow up, that the rapid development of brain atrophy determined substantial cognitive decline, were strengthened also by the partial correlation and multiple regression analyses results which showed that the change in cognitive performances over 2 years was only and independently predicted by the decrease of brain parenchymal volume.

As previously reported by other authors, ${ }^{5}{ }^{19-21}$ in our longitudinal study we did not find any significant relation between overall cognitive impairment and clinical variables such as disability, relapse rate, or duration of disease.

We showed that over 2 years the patients worsened in all MRI parameters, but that the progression was more evident for the loss of brain parenchymal volume, although we detected a statistically significant increase also for T2 and T1 lesion loads. Seven patients had negative T2 lesion volume change at completion of the study. The mean increase of $29.8 \%$ in T2 lesion volume at the end of the follow up is higher than that reported in previous MRI serial studies ${ }^{58}$ and the data from a recently published CHAMPS study ${ }^{59}$ reported, in the placebo group, a median percentage change of T2 lesion volume around $17 \%$ during the 18 month follow up. The differences in duration of disease and in the duration of observation between these studies and ours may perhaps explain the different results. In table 2 we reported that the mean rate of brain atrophy was $1.33 \% / y e a r$. This rate was slightly higher 
than those reported recently by other authors ${ }^{31-33}$ indicating that brain atrophy could be one of the primary pathological events in early disease development and progression.

Other studies performed recently with different MRI and pathological techniques which explored the normal appearing brain tissue pointed out that microscopic diffuse brain damage could be responsible in part for the development of the physical disability. ${ }^{24-31}$ Over 2 years our patient population showed sustained progression of disability. In the patients who deteriorated by one or more points in the EDSS (10 patients) over 2 years, the loss of brain parenchymal volume was pronounced. Moreover, the partial correlation and multiple regression analyses showed a relation between brain parenchymal volume change and EDSS change over the follow up period. These findings disagree with the data recently published by Gee et $a l^{22}$ and by Fox et al. ${ }^{33}$ These authors found a poor correlation between the change in EDSS and the rate of atrophy over 1 year, and speculated that probably such a period was not long enough to allow the demonstration of an association between increasing brain atrophy and changes of EDSS.

Although it is clear that the axonal loss and the demyelination contribute predominantly ${ }^{24}$ to the loss of brain parenchyma in the advanced phases of the disease, it is still unsettled which of the white or grey matter components are firstly involved in the early stages and to what degree. Our study results suggest that the strong relation found after 2 years between neurological impairment and loss of brain parenchyma could be explained early in disease development by the same pathological substrate: the axonal damage which leads irreversibly to neuronal drop out. These data are also supported by the findings of partial correlation analysis, which showed significant correlation coefficients between brain parenchymal volume and $\mathrm{T} 2$ and $\mathrm{T} 1$ lesion loads at baseline, which became more robust at the end of the study. The change in $\mathrm{T} 1$ lesion load (a direct marker of axonal loss) was the only variable along with the EDSS, which independently predicted the change in brain atrophy. Probably, a 1 year period was not long enough to point out an association between progressive cerebral atrophy and change in EDSS, as suggested in previous studies. ${ }^{3133}$

The recent findings of irreversible axonal transection early in the course of multiple sclerosis provided further evidence supporting the importance of early treatment. Use of disease modifying therapy for multiple sclerosis has a demonstrable slowing effect on dysfunction. ${ }^{60}$ Rudick et $a l^{61}$ demonstrated that patients with relapsing-remitting disease on interferon $\beta$-1a therapy had a significant reduction in the progression of brain atrophy during the second year of treatment. Our results indicate that the loss of brain parenchyma in the early stages of relapsing-remitting multiple sclerosis is one of the essential factors responsible for progression of physical disability and cognitive deterioration. Therefore, the early use of disease modifying therapy in clinically asymptomatic patients without remarkable physical disability, but presenting subtle cognitive changes could be reasonable.

In conclusion, we suggest that in the early phase of relapsing-remitting multiple sclerosis cognitive deterioration relies more on the development of brain parenchymal atrophy than on the extent of disease burden in the brain. The loss of brain parenchymal volume underlies the progressive accumulation of physical disability since the initial phase of the disease, and becomes evident only if studied with longer observation periods. Probably, the brain atrophy is caused by the diffuse and early axonal loss, which reflects the progression of neurological deficits and the development of cognitive impairment. These data support the debated opinion that the disease modifying therapy should be initiated as early as possible.

This study has been supported by a grant of the University of Trieste (MURST 60\% R 1996 2/09/01 code 271).

1 Simon JH, Jacobs LD, Campion M, et al. Magnetic resonance studies of intramuscular interferon $\beta-1$ a for relapsing multiple sclerosis. Ann Neurol 1998;43:79-87.

2 Li DKB, Paty DW, the UBC MS/MRI Analysis Research Group and the PRISMS Study Group. Magnetic resonance imaging results of the PRISMS trial: a randomized, double-blind, placebo-controlled study of interferon $\beta-1 \mathrm{~b}$
in relapsing-remitting multiple sclerosis. Ann Neurol 1999; in relapsing-r.

3 The IFNB Multiple Sclerosis Study Group and the University of British Columbia MS/MRI Analysis Group. Interferon $\beta-1 \mathrm{~b}$ in the treatment of multiple sclerosis: final outcome of the randomized controlled trial. Neurology 1995;45:1277-85.

4 Miller DH, Molyneaux PD, Barker GJ, et al. and the European Study Group on interferon $\beta-1 \mathrm{~b}$ in secondary progressive multiple sclerosis. Ann Neurol 1999;46:850-9. psychological assessment and magnetic resonance imaging analysis in multiple sclerosis Arch Neurol 1997;54:1018-25.

6 Miki Y, Grossman RI, Udupa JK, et al. Isolated U-fiber involvement in MS. Preliminary observation. Neurology 1998,50:1301-6.

7 Camp SJ, Stevenson VL, Thompson AJ, et al. Cognitive function in primary progressive and transitional progres-
sive multiple sclerosis. A controlled study with MRI correlates. Brain 1999;122:1341-8.

8 Comi G, Rovaris M, Falautano M, et al. A multiparametric MRI study of frontal lobe dementia in multiple sclerosis. $\mathcal{F}$ Neurol Sci 1999;171:135-44.

9 Filippi M, Tortorella C, Rovaris M, et al. Changes in the normal appearing brain tissue and cognitive impairment in multiple sclerosis. $\mathcal{F}$ Neurol Neurosurg Psychiatry 2000;68: 157-61.

10 Rovaris M, Filippi M, Falautano M, et al. Relation between MR abnormalities and patterns of cognitive impairment in multiple sclerosis. Neurology 1998;50:1601-8. 11 van Buchem MA, Grossman RI, Armstrong C, et al. Correclinical data in MS. Neurology 1998;50:1609-17.

12 Foong J, Rozewicz L, Chong WK, et al. A comparison of neuropsychological deficits in primary and secondary pro-

gressive multiple sclerosis f Neurology 2000;247:97-101.
Rovaris M, Filippi M, Minicucci L, et al. Cortical/

Rovaris M, Filippi M, Minicucci L, et al. Cortical/
subcortical disease burden and cognitive impairment in patients with multiple sclerosis. AfNR Am $\mathcal{f}$ Neuroradiol 2000;21:402-8.

14 Rovaris M, Filippi M. MRI correlates of cognitive dysfunction in multiple sclerosis patients. I Neurovirol 2000;2: S172-5

15 Foong J, Phill M, Rozewicz L, et al. Correlates of executive function in multiple sclerosis. F Neuropsychiatry Clin Neurosci 1999;11:45-50.

16 Blikenberg M, Rune K, Jensen CV, et al. Cortical cerebral metabolism correlates with MRI lesion load and cognitive dysfunction in MS. Neurology 2000;54:558-64.

17 Moriarty DM, Blackshaw JA, Talbot PR, et al. Memory dysfunction in multiple sclerosis corresponds to juxtracortical lesion load on fast fluid-attenuated inversion recovery MR images. AfNR Am $\mathcal{F}$ Neuroradiol 1999;20:1956-62.

18 Fulton JC, Grossman RI, Udupa J, et al. MR lesion load and cognitive function in patients with relapsing-remitting mulcognitive function in patients with relapsing-remitting mul-
tiple sclerosis. AfNR Am f Neuroradiol 1999;20:1951-5.

19 Mariani C, Farina E, Cappa SF, et al. Neuropsychological assessment in multiple sclerosis: a follow up study with magnetic resonance imaging. F Neurol 1991;238:395-400. 
20 Mattioli F, Cappa SF, Cominelli C, et al. Serial study of neuropsychological performance and gadolinium-

Feinstein A, Ron M, Thompson A. A serial study of psycho-
, metric and magnetic resonance imaging in multiple sclerosis. Brain 1993;116:569-602.

22 Aikens JE, Fischer JS, Namey M, et al. A replicated prospective investigation of life stress, coping and depressive symp toms in multiple sclerosis. F Behav Med 1997;20:433-45.

23 Comi G, Martinelli V, Locatelli T, et al. Neurophysiologica and cognitive markers of disease evolution in multiple sclerosis. Multiple Sclerosis 1998;4:260-5.

24 Ferguson B, Matyszak MK, Esiri MM, et al. Axonal damage in acute multiple sclerosis lesions. Brain 1997;120:393-9.

25 Trapp BD, Peterson J, Richard BS, et al. Axonal transection in the lesions of multiple sclerosis. N Eng F Med 1998;338: 278-85.

26 Filippi M, Ianucci G, Tortorella C, et al. Comparison of clinical phenotypes using conventional and magnetization clinical phenotypes using conventional and
transfer MRI. Neurology 1999;52:588-94.

27 Richert NA, Frank JA. Magnetization transfer imaging to monitor clinical trials in multiple sclerosis. Neurology 1999 53:S29-32.

28 Kito M, Goodkin DE, Bacchetti P, et al. Magnetization transfer ratio in new MS lesions before and during therapy with IFN- $\beta$-1a. Neurology 2000;54:1741-5.

29 Barkhof F, van Walderveen $M$. Characterization of tissue damage in multiple sclerosis by nuclear magnetic resonance. Philos Trans R Soc Lond B Biol Sci 1999;354:167586.

30 Mastronardo G, Rocca MA, Ianucci G, et al. A longitudinal MR study of the presymptomatic phase in a patient with clinically definite multiple sclerosis. AfNR Am f Neuroradiol 1999;20:1268-72.

31 Brex PA, Gomez-Ansa B, Parker GJ, et al. Proton MR spectroscopy in clinically isolated syndromes suggestive of multiple sclerosis. F Neurol Sci 1999;166:16-22.

32 Gee Y, Grossman RI, Udupa JK, et al. Brain atrophy in relapsing remitting multiple sclerosis and secondary relapsing remitting multiple sclerosis and secondary progressive multiple sclerosis: long

33 Fox NC, Jenkins R, Leary SM, et al. Progressive cerebral atrophy in MS. A serial study using registered, volumetric MRI Neurology 2000;54:807-12.

34 Inglese M, Rovaris M, Giacomotti L, et al. Quantitative brain volumetric analysis from patients with multiple sclerosis: a follow-up study. $\mathcal{F}$ Neurol Sci 1999;171:8-10.

35 Loseff NA, Wang L, Lai HM, et al. Progressive cerebral atrophy in multiple sclerosis. A serial MRI study. Brain 1996;119:2009-19.

36 Poser CM, Paty DW, Scheinberg L, et al. New diagnostic criteria for multiple sclerosis: guidelines for research protocols. Ann Neurol 1983;12:227-31.

37 Folstein M, Folstein S, Hugh P. Mini mental state: a practical method for grading the cognitive state of patients for clinician. F Psychiatr Res 1975;12:189-98.

38 Raven JC. Guide to the standard progressive matrices. London: HK Lewis, 1960

39 Kurtzke JF. Rating neurologic impairment in multiple sclerosis: an expanded disability status scale (EDSS). Neusclerosis: an expanded dis
rology 1983;33:1444-52.

40 Lublin FD, Reingold SC. Defining the clinical course of multiple sclerosis: results of an international survey. Neurol ogy 1996;46:907-11.
41 FIM Version 4.0. Guide for the uniform data set for medical rehabilitation (Adult-FIM), version 4.0. Buffalo, NY: State rehabilitation (Adult-FIM), version 4.0. Buf

42 Gronwell DMA. Paced auditory serial-addition task: a measure of recovery from concussion. Percept Mot Skills 1977;44:367-73.

43 Stroop JR. Studies of interference in serial verbal reactions. Fxp Psychol 1935;18:643-62.

44 Wechsler D. Manual for the Wechsler adult intelligence scale-revised. New York: Psychological Corporation, 1981.

45 Paradis M, Baruzzi A, Canzanella MA. Test per l'afasia in un bilingue. Hillsdale, NY: Erlbaum, 1990.

46 Patti F, Failla G, Ciancio MR, et al. Neuropsychological, neuroradiological and clinical findings in multiple sclerosis. A 3 year follow-up study. Eur f Neurol 1998;5:283-6.

47 Beatty WW, Goodkin DE. Screening for cognitive impairment in multiple sclerosis. An evaluation of the mini men tal state examination. Arch Neurol 1990;47:297-301.

48 Rao SM, Leo GJ, Bernardin L, et al. Cognitive dysfunction in multiple sclerosis: frequency, patterns and prediction. Neurology 1991;41:685-91.

49 Spinnler H, Tognoni G. Standardizzazione e taratura italiana di test neuropsicologici. Milan: Masson, 1987.

50 Amato MP, Ponziani G, Pracucci G, et al. Cognitive impairment in early onset multiple sclerosis. Pattern, predictors, and impact on everyday life in a 4 year follow up. Arch Neurol 1995;52:168-72.

51 Feinstein A, Youl B, Ron MA. Psychometric, psychiatric, and MRI abnormalities in acute optic neuritis. Brain 1992; 115:1403-15.

52 Hamilton M. Development of a rating scale for primary depressive illness. Br F Soc Clin Psychol 1967;6:278-96.

53 Hamilton $M$. The assessment of anxiety states by rating. $\mathrm{Br}$ f Med Psychol 1959;32:50.

54 Miller DH, Barkhof F, Berry I, et al. Magnetic resonance imaging in monitoring the treatment of multiple sclerosis: concerted action guidelines. 7 Neurol Neurosurg Psyhiatry 1991;54:683-8.

55 Grimaud J, Lai M, Thorpe JW, et al. Quantification of MRI lesion load in multiple sclerosis: a comparison of three computer-assisted techniques. Magn Reson Imaging 1996; 14:495-505.

56 Rovaris M, Filippi M, Calori G, et al. Intra-observer reproducibility in measuring new MR putative markers of demyelination and axonal loss in multiple sclerosis: a comparison with conventional T2-weighted images. F Neurol 1997; 44:266-70.

57 McCarthy K, Warrington E. Cognitive neuropsychology: a clinical introduction. Orlando, FL: Academic Press, 1990

58 Sailer M, O'Riordan JI, Thompson AJ, et al. Quantitative MRI in patients with clinically isolated syndromes suggestive of demyelination. Neurology 1999;52:599-606.

59 Jacobs LD, Beck RW, Simon JH, et al. Intramuscular interferon $\beta$-1 a therapy initiated during a first demyelinating event in multiple sclerosis. N Engl f of Med 2000;343:89890.

60 Fischer JS, Priore R, Jacobs L, et al. Neuropsychological effects of Avonex (interferon $\beta-1 \mathrm{a}$ ) in relapsing multiple sclerosis. Neurology 1998;50:832.

61 Rudick RA, Fisher E, Lee JC, et al. and the Multiple Collaborative Research Group. Use of the brain parenchymal fraction to measure whole brain atrophy in relapsingremitting MS. Neurology 1999;53:1698-704. 